\title{
Mycosis fungoides of the vulva
}

\author{
(D) Wiebren A. A. Tjalma, ${ }^{1,2}$ (D) Michael Janssens, ${ }^{3}$ (D) Karen Dam, ${ }^{1}$ (D) Omar Najim, ${ }^{1}$ (D) Ann Van de Velde, ${ }^{3}$ \\ D Manon Huizing, ${ }^{2,4}$ (D) Zwi Berneman, ${ }^{2,3}$ (D) Wilfried Schroyens ${ }^{2,3}$ \\ ${ }^{1}$ Division Gynaecologic Oncology, Department of Obstetrics and Gynaecology, Multidisciplinary Breast Clinic, Antwerp University Hospital, \\ Antwerp, Belgium \\ ${ }^{2}$ Faculty of Medicine and Health Sciences, University of Antwerp, Antwerp, Belgium \\ ${ }^{3}$ Department of Haematology, Antwerp University Hospital, Antwerp, Belgium \\ ${ }^{4}$ Biobank, Antwerp University Hospital, Antwerp, Belgium
}

\begin{abstract}
Mycosis fungoides (MF) is an indolent form of non-Hodgkin lymphoma and the most common type of primary cutaneous T-cell lymphoma. The overall incidence of MF is approximately 4 per 1 million. Involvement of the vulva by MF is extremely rare, with only seven reported cases in the literature. At the vulva, it is mainly a metastatic lesion and rarely a primary malignancy. We describe a case of vulvar MF and discuss the previous cases. The presentation can easily be confused with benign skin disorders. A vulvar lesion can reflect a systemic disease. When a patient consults for a vulvar lesion it is therefore important not only to look at the vulva but also to examine her in and ask general questions. In a patient with a vulvar mass and cutaneous lesions on other locations MF should be considered in the differential diagnosis.
\end{abstract}

Keywords: Cutaneous T-cell lymphoma; mycosis fungoides; non-Hodgkin lymphoma; vulva.

Cite this article as: Tjalma WAA, Janssens M, Dam K, Najim O, Van de Velde A, Huizing M, et al. Mycosis fungoides of the vulva. North Clin Istanb 2021;8(1):101-105.

$\mathrm{N}_{\mathrm{f}}^{\mathrm{o}}$ on-Hodgkin lymphoma (NHL) may involve the female genital tract, often as a manifestation of systemic disease [1]. Mycosis fungoides (MF) or Alibert-Bazin syndrome, is an indolent form of NHL and the dominant component of primary cutaneous T-cell lymphoma (CTCL) [2, 3].

The overall incidence of MF is rare with only four cases per million [4]. Vulva MF is exceptionally mentioned in the literature. It is difficult to diagnose the disease because it mimics several benign skin disorders. It is therefore likely that vulva MF is under diagnosed. Underreporting could also be present because biopsies are generally not taken at the vulva but rather at other easily accessible skin locations. A Medline search August $10^{\text {th }}$ 2018, with the terms vulva and mycosis fungoides revealed that there were only seven published cases $[2,3,5-7]$. The present case study will describe an additional case together with a review of the previous published cases.

\section{CASE REPORT}

A 58-year old woman, G1P1, consulted the outpatient clinic with a 4-week history of itching and a painless nodule in the right labium majus. Previous antibiotic treatment did not affect. At that moment, she received treatment with gemcitabine (400 mg per week intravenously) for mycosis fungoides (MF). The MF was diagnosed five years earlier after three years of complaints. The patient also suffered from hypothyroidism and hypertension. Since, one year she was menopausal. 
Her previous treatments for MF included PUVA and UVB (discontinued due to intolerance), interferon (discontinued due to liver function failure), bexarotene (discontinued due to multiple side effects), methotrexate, carmustine (discontinued due to contact dermatitis), topical corticoids and adjuvant radiotherapy.

On clinical examination, there was a red, hard, but painless nodule $(3 \mathrm{~cm}$ ) in the right labium majus (Fig. 1A). Lateral from the enlarged labium there was also a demarcated, hypopigmented or white patch. This lesion was suggestive for hypopigmented MF. On her forearm she had a patch with erythema and mild scale (Fig. 1B). The vulva nodule was excised, and the pathological assay showed that there was a dermal infiltration of small-cell lymphocytes, positive for CD3 on an immunohistochemical assay, confirming the diagnosis of MF. The patient continued to receive gemcitabine. A CHOP-scheme (cyclophosphamide, doxorubicin, vincristine and prednisone) was initiated four months later. This caused neutropenic fever, for which hospitalization and antibiotics were needed. Despite the combined cy tostatic treatment, new tumor lesions appeared. Total Skin Electron Beam (TSEB) was initiated two months later for a period of five weeks. Four months after TSEB she received a haploidentical stem cell transplantation. Unfortunately, this was complicated by a graft-versus-host disease, neutropenic fever and hemodynamic instability. The patient deceased 13 months after the diagnosis of vulva MF.

\section{DISCUSSION}

The vulva can be involved by lymphoma's mostly secondary and sometimes primarily [1]. MF is the most common cutaneous T-cell lymphoma. MF still has an unclear etiology. The mean age for patients at the time of MF diagnosis is between 40 and 60 years [4] and it is more common in men then in women $(2: 1$ male: female ratio) $[8,9]$.

Although MF has a predilection for sun-protected areas (bathing trunk distribution), they are uncommon in the vulva region. Typical areas for MF are the chest, inner arms, lower trunk, groin and buttocks [7]. A literature search revealed that there were only seven published cases of vulva MF [2, 3, 5-7]. In Table 1, current case together with the published cases are shown. In only one case, MF was a primary malignancy [6], in all the other cases, it had to be considered as a manifestation of an advanced stage of MF. In one case, it looked like a primary but the past medical history suggested that the patient had MF already for eight years on her breast [3].
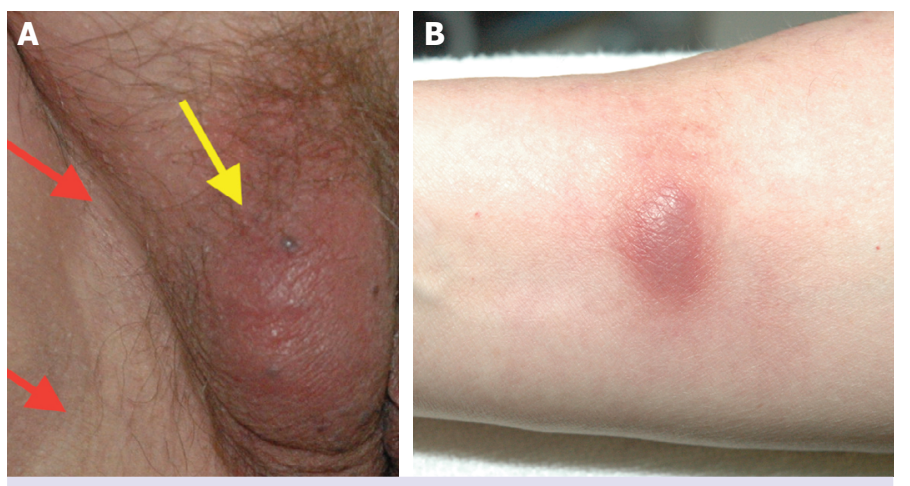

FIGURE 1. (A) Vulva MF: A dark red area and mass in the right labium majus (yellow arrow). Lateral from the labium, there is a hypopigmented lesion (red arrows). (B) Typical MF patch with erythema and mild scale on the forearm.

All cases of vulva MF were located on the labia. In five cases, there was a mass, in one case, only an enlargement of the right labia ( $\mathrm{x} 5)$, in one case, only pruritus and in one case of pruritus and a chronic fissure. In general, MF is characterized by progressive highly symptomatic skin lesions, including patches, plaques, tumors, and erythroderma [10]. Erythroderma, defined as $>80 \%$ body surface involvement with patches and/or plaques, is associated with pruritus, leonine facies, skin atrophy, lichenification, and hyperkeratosis of the palms and soles [10]. The diagnosis of MF in general is difficult due to the non-specific skin lesions and the non-diagnostic biopsies. The patient with a chronic vulva fissure and MF, had in retrospect already MF on her breast eight years earlier [3]. It was only by assuming a possible clinical link between the two locations and repeating the biopsies that MF was diagnosed [3]. A delay in diagnosis or a misdiagnosis in MF is not uncommon. All women with a metastatic lesion at the vulva had skin lesions at other locations. Therefore, it is important to examine the patient detailed and ask her general questions when she consults for a vulvar dermatosis. When there are lesions at other locations and there is a possible clinical link one should take (new) biopsies at all locations. The differential diagnosis of MF lesions at the vulva are extensive and include very common infections, autoimmune diseases and rare malignant diseases (e.g., candida, herpes, intertrigo, eczema, lichen sclerosus, lichen planus, lichen simplex chronicus, psoriasis, Paget's disease, verrucous carcinoma, epithelioid sarcoma, myxoid leiomyosarcoma) [11-14].

The diagnostic algorithm for early MF is based on clinical, histopathologic, molecular/biologic and immunological criteria [15]. MF is staged according to 


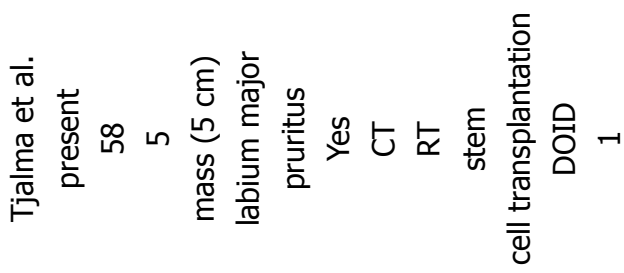

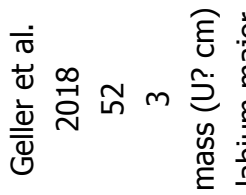

$\stackrel{0}{x}$

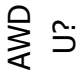

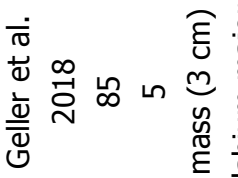

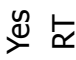

กิจ

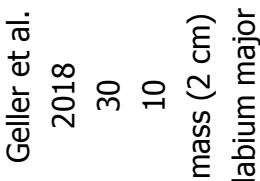

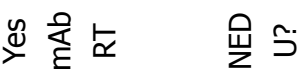

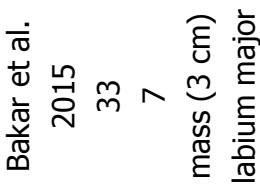

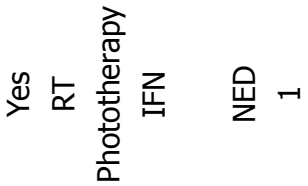

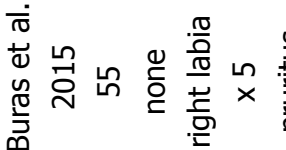

을 은 $\frac{7}{\frac{1}{2}}$

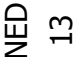

它

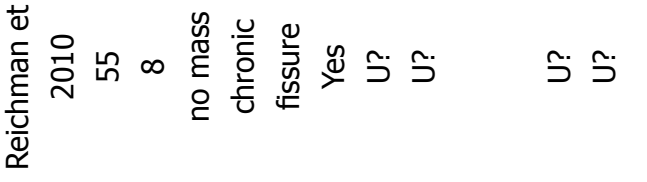

$\frac{1}{2} \frac{10}{2}$

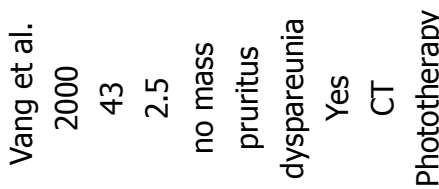
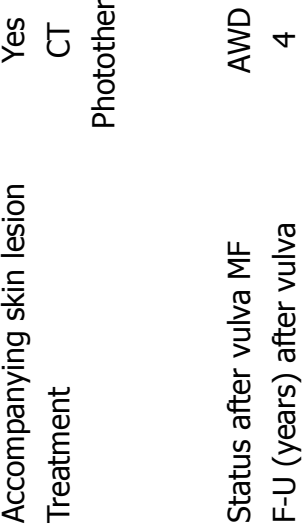

the $\mathrm{T}$ (skin), $\mathrm{N}$ (lymph nodes), $\mathrm{M}$ (viscera) and $\mathrm{B}$ (blood) classification [16]. This TNMB classification stratifies patients into those with early-stage (stage IA to IIA) or advanced stage (stage IIB to IVB) disease $[16,17]$.

The treatment is based on the skin manifestations and the systemic disease. Patients with skin-limited disease respond well on skin directed therapies. Local therapy alone in these circumstances can produce remission and even cure. The local therapies can consist of topical chemotherapy (e.g., mechlorethamine or nitrogen mustard, carmustine), topical rexinoids (e.g., bexarotene), topical steroids, topical imiquimod, phototherapy [= Ultraviolet light, including UVB (wavelength $320-290 \mathrm{~nm}$ ), narrow band UVB (wavelength $311 \mathrm{~nm}$ ), and psoralen plus UVA (PUVA, wavelength $400-320 \mathrm{~nm}$ )] and radiotherapy (locally to a single lesion or group of lesions or to the entire skin surface via TSEBT (total skin electron beam therapy (TSEBT) $[4,10$, 18]. Patients with extensive plaques and patches should receive intensive topical therapy to induce a complete remission followed by less intensive adjuvant topical therapy to sustain remission [10]. For patients with more advanced disease (tumors, erythroderma, nodal, visceral, or blood disease), curation is limited and the treatment should include cutaneous symptom palliation and systemic disease control [10]. Systemic therapy can consist of immunomodulator therapy (e.g., interferon), retinoids (e.g., isotretinoin, acitretin), rexinoids (e.g., bexarotene), denileukin diftitox, histone deacetylase (HDAC) inhibitors (e.g., vorinostat), extracorporeal photochemotherapy (ECP), systemic chemotherapy, allogeneic stem cell transplantation and emerging therapies (e.g., pralatrexate, cytokines, enhancer of $T$ helper 1 cellular immunity (e.g., lenalidomide), proteosome inhibitor (e.g., bortezomib), purine nucleoside phosphorylase inhibitor (e.g., forodesine), in situ vaccination (intratumoral injection of a TLR9 agonist) combined with radiation and monoclonal antibody therapies (e.g., alemtuzumab, zanolimumab, brentuximab vedotin) $[4,10,19,20]$. The participation of course in clinical trials, like always, should be stimulated.

The survival of MF depends on the stage. MF Patients limited patch and plaque (clinical stage IA) do not have an altered life expectancy and less than $10 \%$ will progress to more advanced stages [21]. The 
median survival of MF patients with generalized patch and/or plaque (T2) is 11.7 years [22]. In this stage, $24 \%$ will experience disease progression to a more advanced clinical stage and approximately $20 \%$ eventually die of the disease [22]. In advanced MF (stage IIB to IV; tumors and erythroderma) the median OS is 63 months, with 2 - and 5 -year survival rates of $77 \%$ and $52 \%$, respectively [17].

Except for one case, vulvar involvement of MF was a sign of disease progression and therefore associated with a poor prognosis. Partial and sometimes complete local remission could be achieved in these cases with local therapies. Radiotherapy for MF is in general very effective for early and advanced stage MF. Due to the tenderness of the vulva, it seems better to administer local radiotherapy in a low dose in multiple fractions (e.g., $4 \times 2$ Gy) instead of a single high dose of $8 \mathrm{~Gy}$ [2]. Exceptional is the case of primarily vulva MF with surgery as the sole treatment [6]. In this patient, the right labia minora et majora were five times the normal size. The skin was unremarkable for ulcerations, scaling plaques or microabscesses. She underwent a right hemivulvectomy with bilateral groin lymph node dissection. No adjuvant therapy was administered, and 13 years after the surgery, the patient is well with no evidence of recurrence [6].

\section{Conclusion}

Vulva MF is a diagnostic and therapeutic challenge. When confronted with a vulva dermatosis, a careful anamnesis and evaluation for a primary site elsewhere should be performed. The appearance can mimic benign skin diseases, and biopsies can be a false negative, causing a delayed diagnosis. It is important to repeat biopsies in case of the persistent lesion(s) with an unclear diagnosis. The approach seems aggressive, but it is the only way to reduce morbidity. Vulvar MF should be considered a sign of progressive disease with general a poor prognosis. The treatment should be tailored according to the symptoms and the extent of the disease. Local remission (partial or complete) can be achieved by local therapies.

Informed Consent: Written informed consent was obtained from the patient for the publication of the case report and the accompanying images.

Conflict of Interest: No conflict of interest was declared by the authors.

Financial Disclosure: The authors declared that this study has received no financial support.
Authorship Contributions: Concept - WAAT, MH, ZB, WS; Design WAAT, MJ, KD, ON, AVV, MH, ZB, WS; Supervision - WAAT; Materials - WAAT, MJ, KD, ON, AVV, MH, ZB, WS; Data collection and/or processing - WAAT, MJ, KD, ON, AVV, MH, ZB, WS; Analysis and/or interpretation - WAAT, MJ, KD, ON, AVV, MH, ZB, WS; Literature review - WAAT, $M J, K D$, ON, AVV, MH, ZB, WS; Writing - WAAT, MJ, KD, ON, AVV, MH, ZB, WS; Critical review - WAAT, MJ, KD, ON, AVV, MH, ZB, WS.

\section{REFERENCES}

1. Tjalma WA, Van de Velde AL, Schroyens WA. Primary non-Hodgkin's lymphoma in Bartholin's gland. Gynecol Oncol 2002;87:308-9.

2. Bakar Ö, Şahin S, Çetinözman F, Willemze R, Tezcanli E, Çetin ED. Tumor-stage mycosis fungoides of the vulva successfully treated with local low-dose radiotherapy. Dermatol Ther 2015;28:36-9. [CrossRef]

3. Reichman O, Sobel JD, Bentley G. Chronic vulvar fissure--a rare manifestation of mycosis fungoides. J Low Genit Tract Dis 2010;14:65-7.

4. Foss FM, Girardi M. Mycosis Fungoides and Sezary Syndrome. Hematol Oncol Clin North Am 2017;31:297-315. [CrossRef]

5. Vang R, Medeiros LJ, Malpica A, Levenback C, Deavers M. NonHodgkin's lymphoma involving the vulva. Int J Gynecol Pathol 2000;19: 236-42. [CrossRef]

6. Buras AL, McSwain AN, Qin Y, Meunier JK. Primary mycosis fungoides of the vulva: The first reported case. Gynecol Oncol Rep 2015;12:7-8. [CrossRef]

7. Geller S, Pitter K, Moskowitz A, Horwitz SM, Yahalom J, Myskowski PL. Treatment of Vulvar Mycosis Fungoides Tumors With Localized Radiotherapy. Clin Lymphoma Myeloma Leuk 2018;18:e279-81.

8. Sant M, Allemani C, Tereanu C, De Angelis R, Capocaccia R, Visser $\mathrm{O}$, et al; HAEMACARE Working Group. Incidence of hematologic malignancies in Europe by morphologic subtype: results of the HAEMACARE project. Blood 2010;116:3724-34. [CrossRef]

9. Criscione VD, Weinstock MA. Incidence of cutaneous T-cell lymphoma in the United States, 1973-2002. Arch Dermatol 2007;143:854-9.

10. Galper SL, Smith BD, Wilson LD. Diagnosis and management of mycosis fungoides. Oncology (Williston Park) 2010;24:491-501.

11. Tjalma WA, Hauben EI, Deprez SM, Van Marck EA, van Dam PA. Epithelioid sarcoma of the vulva. Gynecol Oncol 1999;73:160-4.

12. Tjalma WA. Extramammary Paget's disease of the vulva. J Am Coll Surg 2003;197:701-2. [CrossRef]

13. Tjalma WA, Siozopoulou V, Huizing MT. A clitoral verrucous carcinoma in an area of lichen planus has aggressive features. World J Surg Oncol 2017;15:7. [CrossRef]

14. Tjalma WA, Colpaert CG. Myxoid leiomyosarcoma of the vulva. Gynecol Oncol 2005;96:548-51. [CrossRef]

15. Pimpinelli N, Olsen EA, Santucci M, Vonderheid E, Haeffner AC, Stevens $\mathrm{S}$, et al; International Society for Cutaneous Lymphoma. Defining early mycosis fungoides. J Am Acad Dermatol 2005;53:1053-63.

16. Olsen E, Vonderheid E, Pimpinelli N, Willemze R, Kim Y, Knobler $\mathrm{R}$, et al; ISCL/EORTC. Revisions to the staging and classification of mycosis fungoides and Sezary syndrome: a proposal of the International Society for Cutaneous Lymphomas (ISCL) and the cutaneous lymphoma task force of the European Organization of Research and Treatment of Cancer (EORTC). Blood 2007;110:1713-22. [CrossRef]

17. Scarisbrick JJ, Prince HM, Vermeer MH, Quaglino P, Horwitz S, Porcu P, et al. Cutaneous Lymphoma International Consortium Study of Outcome in Advanced Stages of Mycosis Fungoides and Sézary Syndrome: Effect of Specific Prognostic Markers on Survival and Development of a Prognostic Model. J Clin Oncol 2015;33:3766-73. [CrossRef] 
18. Hoppe RT, Harrison C, Tavallaee M, Bashey S, Sundram U, Li S, et al. Low-dose total skin electron beam therapy as an effective modality to reduce disease burden in patients with mycosis fungoides: results of a pooled analysis from 3 phase-II clinical trials. J Am Acad Dermatol 2015;72:286-92. [CrossRef]

19. Kim YH, Gratzinger D, Harrison C, Brody JD, Czerwinski DK, Ai $\mathrm{WZ}$, et al. In situ vaccination against mycosis fungoides by intratumoral injection of a TLR9 agonist combined with radiation: a phase 1/2 study. Blood 2012;119:355-63. [CrossRef]

20. Kim YH, Tavallaee M, Sundram U, Salva KA, Wood GS, Li S, et al.
Phase II Investigator-Initiated Study of Brentuximab Vedotin in Mycosis Fungoides and Sézary Syndrome With Variable CD30 Expression Level: A Multi-Institution Collaborative Project. J Clin Oncol 2015;33:3750-8. [CrossRef]

21. Kim YH, Jensen RA, Watanabe GL, Varghese A, Hoppe RT. Clinical stage IA (limited patch and plaque) mycosis fungoides. A long-term outcome analysis. Arch Dermatol 1996;132:1309-13. [CrossRef]

22. Kim YH, Chow S, Varghese A, Hoppe RT. Clinical characteristics and long-term outcome of patients with generalized patch and/or plaque (T2) mycosis fungoides. Arch Dermatol 1999;135:26-32. [CrossRef] 